\title{
CONFIRMATION OF $\mathrm{C}_{60}$ IN THE REFLECTION NEBULA NGC 7023
}

\author{
K. Sellgren ${ }^{1}$, M.W. Werner ${ }^{2}$, J.G. Ingalls ${ }^{3}$, J.D.T. Smith ${ }^{4}$, \\ T.M. Carleton ${ }^{5}$ and C. Joblin 6,7
}

\begin{abstract}
The fullerene $\mathrm{C}_{60}$ has four infrared active vibrational transitions at $7.0,8.5,17.5$ and $18.9 \mu \mathrm{m}$. We have previously observed emission features at 17.4 and $18.9 \mu \mathrm{m}$ in the reflection nebula NGC 7023 and demonstrated spatial correlations suggestive of a common origin. We now confirm the identification of these features with $\mathrm{C}_{60}$ by detecting a third emission feature at $7.04 \pm 0.05 \mu \mathrm{m}$ at a position of strong $18.9 \mu \mathrm{m}$ emission in NGC 7023. We also report the detection of these three features in the reflection nebula NGC 2023. We show with spectroscopic mapping of NGC 7023 that the $18.9 \mu \mathrm{m}$ feature peaks on the central star, that the $16.4 \mu \mathrm{m}$ emission feature due to PAHs peaks between the star and a nearby photodissociation front, and that the $17.4 \mu \mathrm{m}$ feature is a blend of a PAH feature and $\mathrm{C}_{60}$. The derived $\mathrm{C}_{60}$ abundance is consistent with that from previous upper limits and possible fullerene detections in the interstellar medium.
\end{abstract}

\section{Introduction}

Fullerenes are cage-like molecules (spheroidal or ellipsoidal) of pure carbon, such as $\mathrm{C}_{60}, \mathrm{C}_{70}, \mathrm{C}_{76}$, and $\mathrm{C}_{84}$. $\mathrm{C}_{60}$ is the most stable fullerene and can account for up to $50 \%$ of the mass of fullerenes generated in the laboratory (Kroto et al. 1985). Foing \& Ehrenfreund (1994) propose that two diffuse interstellar bands at 958 and $963 \mathrm{~nm}$ are due to singly ionized $\mathrm{C}_{60}$, or $\mathrm{C}_{60}^{+}$, although the identification is debated

\footnotetext{
1 Department of Astronomy, Ohio State University

2 Jet Propulsion Laboratory, California Institute of Technology

3 Spitzer Science Center, California Institute of Technology

4 Ritter Astrophysical Research Center, University of Toledo

5 University of Arizona

6 Université de Toulouse, UPS, CESR

7 CNRS
} 
(Maier 1994; Jenniskens et al. 1997). Misawa et al. (2009) attribute additional diffuse interstellar bands at 902,921 , and $926 \mathrm{~nm}$ to $\mathrm{C}_{60}^{+}$. Observational evidence for neutral fullerenes, however, has been elusive to date. No fullerenes have yet been found towards carbon-rich post-AGB stars (Somerville \& Bellis 1989; Snow \& Seab 1989), carbon stars (Clayton et al. 1995; Nuccitelli et al. 2005), or R CrB stars (Clayton et al. 1995; Lambert et al. 2001). Similarly, no neutral fullerenes have yet been found in the diffuse interstellar medium (Snow \& Seab 1989; Herbig 2000), dense molecular clouds (Nuccitelli et al. 2005), or at the photodissociation front in the reflection nebula NGC 7023 (Moutou et al. 1999).

$\mathrm{C}_{60}$ has four infrared-active vibrational transitions, at 7.0, 8.5, 17.4, and $18.9 \mu \mathrm{m}$ (Frum et al. 1991; Sogoshi et al. 2000). We tentatively identified the 17.4 and $18.9 \mu \mathrm{m}$ interstellar emission features in the reflection nebula NGC 7023 as due to $\mathrm{C}_{60}$ (Werner et al. 2004b; Sellgren et al. 2007).

We report here the detection of the $\mathrm{C}_{60}$ feature at $7.04 \pm 0.05 \mu \mathrm{m}$ in NGC 7023 . We also report the detection of $\mathrm{C}_{60}$ features at $7.04,17.4$ and $18.9 \mu \mathrm{m}$ in a second reflection nebula, NGC 2023. The $\mathrm{C}_{60} 8.5 \mu \mathrm{m}$ feature is too blended with the strong $8.6 \mu \mathrm{m}$ PAH feature to yield a useful limit. We have found earlier (Sellgren et al. 2007) that the $18.9 \mu \mathrm{m}$ emission feature in NGC 7023 has a spatial distribution distinct from that of the $16.4 \mu \mathrm{m}$ emission feature attributed to polycyclic aromatic hydrocarbons (PAHs). We now compare the spatial distributions of the $16.4,17.4$, and $18.9 \mu \mathrm{m}$ emission features in NGC 7023, and find additional suppport for the $\mathrm{C}_{60}$ identification.

\section{Results}

We have used the Spitzer Space Telescope (Werner et al. 2004a) with the Infrared Spectrograph (IRS; Houck et al. 2004) to obtain spectra of NGC 7023 and NGC 2023. We used the short-wavelength low-resolution module SL $(5-14 \mu \mathrm{m}$; $\Delta \lambda / \lambda=60-120)$ and the long-wavelength low-resolution module LL2 (14-20 $\mu \mathrm{m}$; $\Delta \lambda / \lambda=60-120)$. Nebular positions were chosen for a strong ratio of the $18.9 \mu \mathrm{m}$ feature relative to the $16.4 \mu \mathrm{m}$ PAH feature. We also made a spectroscopic map with the LL2 module of NGC 7023.

We show our 14-20 $\mu \mathrm{m}$ spectrum of NGC 2023 in Figure 1. We mark the wavelengths of $\mathrm{C}_{60}$ lines at 17.4 and $18.9 \mu \mathrm{m}$, of a PAH feature at $16.4 \mu \mathrm{m}$, and of $\mathrm{H}_{2}$ emission at $17.0 \mu \mathrm{m}$.

We show our 5-9 $\mu \mathrm{m}$ spectra of NGC 7023 in Figure 2. We clearly detect an emission feature at $7.04 \pm 0.05 \mu \mathrm{m}$. We detect the $7.04 \mu \mathrm{m}$ feature also in NGC 2023. This feature is coincident, within the uncertainties, with the wavelength of the expected $\mathrm{C}_{60}$ line. We highlight this emission feature by fitting the 5-9 $\mu \mathrm{m}$ spectrum with a blend of PAH emission features in addition to the new emission feature at $7.04 \mu \mathrm{m}$. We perform this model fit using PAHFIT (Smith et al. 2007a).

In our previous long-slit spectroscopic investigation of NGC 7023 (Sellgren et al. 2007), we found that the $18.9 \mu \mathrm{m}$ feature peaks closer to the central star than either neutral PAHs or ionized PAHs. We now use the LL2 spectroscopic 


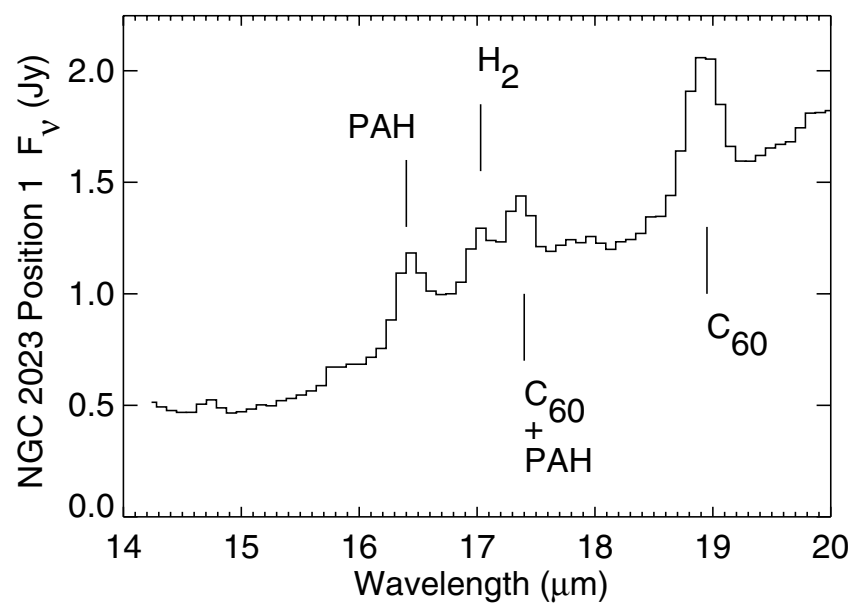

Fig. 1. Spitzer-IRS spectrum of NGC 2023 (solid black histogram), obtained with the long-wavelength low-resolution module (LL2; 14-20 $\mu \mathrm{m} ; \lambda / \Delta \lambda=60-130$ ). We mark the wavelengths of $\mathrm{C}_{60}$ at 17.4 and $18.9 \mu \mathrm{m}$, a PAH feature at $16.4 \mu \mathrm{m}$, and $\mathrm{H}_{2}$ emission at $17.0 \mu \mathrm{m}$ (vertical solid lines).

map extracted in NGC 7023 to illustrate this point more clearly in Figure 3. The $18.9 \mu \mathrm{m}$ emission is clearly centered on the star. By contrast, the $16.4 \mu \mathrm{m}$ PAH emission peaks outside the region of maximum $18.9 \mu \mathrm{m}$ emission, in a layer between the star and the molecular cloud. The photodissociation front at the UV-illuminated front surface of the molecular cloud is delineated by fluorescent $\mathrm{H}_{2}$ emission at $17.0 \mu \mathrm{m}$.

We show an image of the $17.4 \mu \mathrm{m}$ emission from NGC 7023 in Figure 3, overlaid with contours of $18.9 \mu \mathrm{m}$ and $16.4 \mu \mathrm{m}$ emission. The $17.4 \mu \mathrm{m}$ emission clearly shows one peak on the central star, coincident with the $18.9 \mu \mathrm{m} \mathrm{C}_{60}$ emission, and a second peak co-spatial with the $16.4 \mu \mathrm{m}$ PAH emission. We conclude that the observed $17.4 \mu \mathrm{m}$ emission feature is a blend of a PAH feature at $17.4 \mu \mathrm{m}$, whose spatial distribution follows that of the $16.4 \mu \mathrm{m}$ PAH feature, and of $17.4 \mu \mathrm{m} \mathrm{C}_{60}$ emission.

\section{Discussion}

We have detected three lines due to $\mathrm{C}_{60}$, in two reflection nebulae. This is the first detection of neutral $\mathrm{C}_{60}$ in space.

Theorists have suggested that fullerenes might form around stars with carbonrich atmospheres, such as carbon stars, cool carbon-rich Wolf-Rayet (WC) stars, and C-rich, H-poor R Cr B stars (Kroto \& Jura 1992; Goeres \& Sedlmayr 1992; Cherchneff et al. 2000; Pascoli \& Polleux 2000). Fullerenes may also form as 


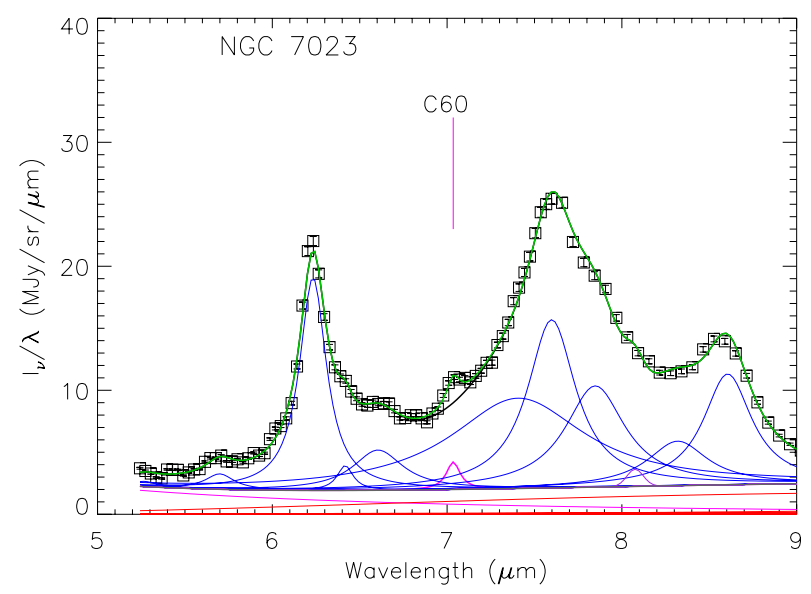

Fig. 2. Spitzer-IRS 5-9 $\mu \mathrm{m}$ spectrum of NGC 7023, obtained with the short-wavelength low-resolution module (SL; $\lambda / \Delta \lambda=60-120$; solid black histogram). The wavelength of $\mathrm{C}_{60}$ at $7.0 \mu \mathrm{m}$ is marked (vertical solid magenta line). We show the individual contributions of $\mathrm{PAH}$ features at 5.3, 5.7, 6.2, 6.4, 6.7, 7.4, 7.6, 7.8, 8.3, and $8.6 \mu \mathrm{m}$ to the spectrum, by decomposing the spectrum with PAHFIT (Smith et al. 2007a) and then overplotting the Lorentzian profile of each feature (thin solid black curves). The Lorentzian fit to the $\mathrm{C}_{60}$ feature we detect at $7.04 \pm 0.05 \mu \mathrm{m}$ is highlighted (thick solid magenta curve).

part of the carbon-rich grain condensation process known to occur in the ejecta of Type II supernovae (Clayton et al. 2001). Fullerenes might form via interstellar gas-phase chemistry in dense and diffuse molecular clouds (Bettens \& Herbst 1996, 1997). Hydrogenated amorphous carbon grains in the interstellar medium may also decompose after interstellar shocks into fullerenes and PAHs (Scott et al. 1997).

We compare the relative amounts of stellar flux absorbed and re-radiated by fullerenes and PAHs to derive the abundance of $\mathrm{C}_{60}$ in NGC 7023 and NGC 2023. First we compare the sum of the intensities of the 7.0,17.4, and $18.9 \mu \mathrm{m}$ features, assumed to be due to $\mathrm{C}_{60}$, to the sum of all other infrared emission features at 5-20 $\mu \mathrm{m}$, assumed to be due to PAHs. We ignore potential visual fluorescence by $\mathrm{C}_{60}$ or PAHs, do not include any potential $8.5 \mu \mathrm{m} \mathrm{C}_{60}$ emission, and do not attempt to correct the $17.4 \mu \mathrm{m} \mathrm{C}_{60}$ emission for any $\mathrm{PAH}$ contribution. We used PAHFIT (Smith et al. 2007a) to find that the ratio of $\mathrm{C}_{60}$ to $\mathrm{PAH}$ emission is 0.01-0.03 in NGC 7023 and NGC 2023. We then compare the amount of UV starlight per $\mathrm{C}$ atom absorbed by $\mathrm{C}_{60}$ (Yasumatsu et al. 1996; Yagi et al. 2009). and PAHs ( $\mathrm{Li} \&$ Draine 2001). We assume that $9-18 \%$ of interstellar carbon is in PAHs (Joblin et al. 1992; Tielens 2008). Our preliminary estimate of the percentage of interstellar carbon contained in $\mathrm{C}_{60}, p\left(C_{60}\right)$, is $0.1-0.6 \%$. 

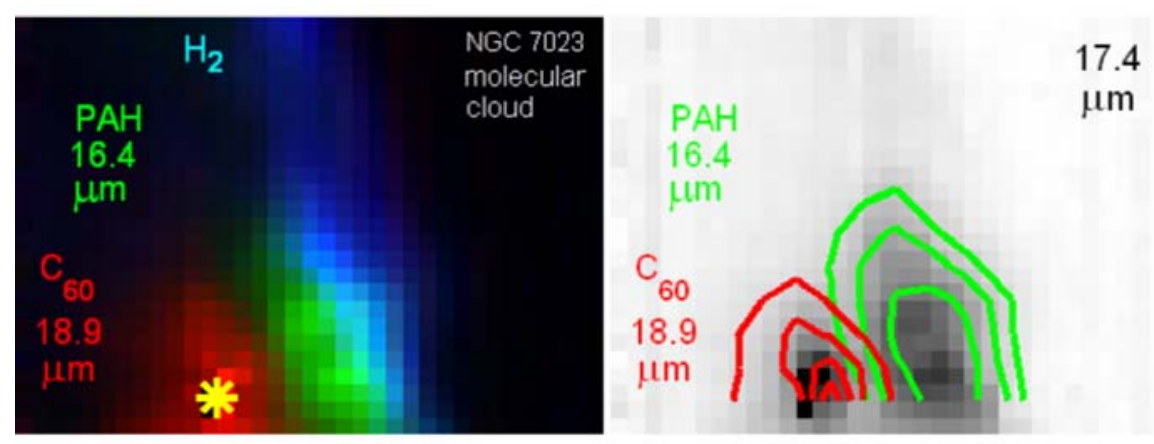

Fig. 3. Spectral map of NGC 7023, obtained with the Spitzer IRS 15-20 $\mu \mathrm{m}$ LL2 longslit module, extracted and analyzed using CUBISM (Smith et al. 2007b). Each pixel is $5.1^{\prime \prime} \times 5.1^{\prime \prime}$. Left: three-color image of NGC 7023, with the $18.9 \mu \mathrm{m} \mathrm{C}_{60}$ feature in red, the $16.4 \mu \mathrm{m}$ PAH feature in green, and the $17.0 \mu \mathrm{m} \mathrm{H}_{2}$ line in blue. The position of the central star is marked with a cross. The $18.9 \mu \mathrm{m} \mathrm{C}_{60}$ feature peaks on the central star. The front surface of the UV-illuminated molecular cloud is traced by $\mathrm{H}_{2}$ emission, while the $16.4 \mu \mathrm{m}$ PAH emission peaks between the $\mathrm{C}_{60}$ and $\mathrm{H}_{2}$ emission. Right: grayscale image of NGC 7023 in the $17.4 \mu \mathrm{m}$ feature, overlaid with contours of $18.9 \mu \mathrm{m} \mathrm{C}_{60}$ emission (red), and contours of $16.4 \mu \mathrm{m} \mathrm{PAH} \mathrm{feature} \mathrm{emission} \mathrm{(green).} \mathrm{The} \mathrm{observed}$ $17.4 \mu \mathrm{m}$ emission has two spatial components, one co-spatial with $\mathrm{C}_{60}$ emission and the other co-spatial with PAH emission. We conclude that the $17.4 \mu \mathrm{m}$ emission feature is due to a blend of PAH and $\mathrm{C}_{60}$ emission.

Our preliminary estimate of $p\left(C_{60}\right)$ is consistent with published estimates of the percentage of carbon in $\mathrm{C}_{60}^{+}$in diffuse clouds $(0.1-0.9 \%$; Foing \& Ehrenfreund 1994; Herbig 2000). It is also consistent with previous upper limits on $p\left(C_{60}\right)$ towards R Coronae Borealis, massive young stellar objects, and NGC $7023(<0.3-$ 0.6\%; Moutou et al. 1999; Nuccitelli et al. 2005).

\section{Conclusions}

We confirm our previous suggestion that emission features at 17.4 and $18.9 \mu \mathrm{m}$ in NGC 7023 are due to $\mathrm{C}_{60}$, by detecting a third $\mathrm{C}_{60}$ line at $7.04 \mu \mathrm{m}$. We detect the $7.04,17.4$, and $18.9 \mu \mathrm{m} \mathrm{C}_{60}$ lines in NGC 2023 also. The spatial distribution of the $17.4 \mu \mathrm{m}$ feature in NGC 7023, compared to the spatial distributions of $16.4 \mu \mathrm{m}$ $\mathrm{PAH}$ and $18.9 \mu \mathrm{m} \mathrm{C}_{60}$ emission, suggests that the observed $17.4 \mu \mathrm{m}$ feature is a superposition of two lines, one due to PAHs and one due to $\mathrm{C}_{60}$. The abundance we derive for $\mathrm{C}_{60}$ is consistent with previous upper limits on $\mathrm{C}_{60}$ and previous measurements of lines identified with $\mathrm{C}_{60}^{+}$. Our observations are the first firm detection of $\mathrm{C}_{60}$ in space.

This work is based on observations made with the Spitzer Space Telescope, which is operated by the Jet Propulsion Laboratory, California Institute of 
Technology under a contract with NASA. Support for this work was provided by NASA through an award issued by JPL/Caltech.

\section{References}

Bettens, R.P.A., \& Herbst, E., 1997, ApJ, 478, 585

Bettens, R.P.A., \& Herbst, E., 1996, ApJ, 468, 686

Cherchneff, I., Le Teuff, Y.H., Williams, P.M., \& Tielens, A.G.G.M., 2000, A\&A, 357, 572

Clayton, D.D., Deneault, E.A.-N., \& Meyer, B.S., 2001, ApJ, 562, 480

Clayton, G.C., Kelly, D.M., Lacy, J.H., et al., 1995, AJ, 109, 2096

Foing, B.H., \& Ehrenfreund, P., 1994, Nature, 369, 296

Frum, C.I., Engleman, R.J., Hedderich, H.G., et al., 1991, Chem. Phys. Lett., 176, 504

Goeres, A., \& Sedlmayr, E., 1992, A\&A, 265, 216

Herbig, G.H., 2000, ApJ, 542, 334

Houck, et al., 2004, ApJS, 154, 18

Jenniskens, P., Mulas, G., Porceddu, I., \& Benvenuti, P., 1997, A\&A, 327, 337

Kroto, H.W., Heath, J.R., O’Brien, S.C., Curl, R.F., \& Smalley, R.E., 1985, Nature, 318, 162

Joblin, C., Léger, A., \& Martin, P., 1992, ApJ, 393, L79

Kroto, H.W., \& Jura, M., 1992, A\&A, 263, 275

Lambert, D.L., Rao, N.K., Pandey, G., \& Ivans, I.I., 2001, ApJ, 555, 925

Li, A., \& Draine, B.T., 2001, ApJ, 554, 778

Maier, J.P., 1994, Nature, 370, 423

Misawa, T., Gandhi, P., Hida, A., Tamagawa, T., \& Yamaguchi, T., 2009, ApJ, 700, 1988

Moutou, C., Sellgren, K., Verstraete, L., \& Léger, A., 1999, A\&A, 347, 949

Nuccitelli, D., Richter, M.J., \& McCall, B.J., 2005, IAU Symp., 235, 236

Pascoli, G., \& Polleux, A., 2000, A\&A, 359, 799

Scott, A., Duley, W.W., \& Pinho, G.P., 1997, ApJ, 489, L193

Sellgren, K., Uchida, K.I., \& Werner, M.W., 2007, ApJ, 659, 1338

Smith, J.D.T., et al., 2007a, ApJ, 656, 770

Smith, J.D.T., et al., 2007b, PASP, 119, 1133

Snow, T.P., \& Seab, C.G., 1989, A\&A, 213, 291

Sogoshi, N., Kato, Y., Wakabayashi, T., et al., 2000, J. Phys. Chem. A., 104, 3733

Somerville, W.B., \& Bellis, J.G., 1989, MNRAS, 240, 41P

Tielens, A.G.G.M., 2008, ARA\&A, 46, 289

Werner, M.W., et al., 2004, ApJS, 154, 1

Werner, M.W., et al., 2004, ApJS, 154, 309

Yagi, H., et al., 2009, Carbon, 47, 1152

Yasumatsu, H., Kondow, T., Kitagawa, H., Tabayashi, K., \& Shobatake, K., 1996, J. Chem. Phys., 104, 899 